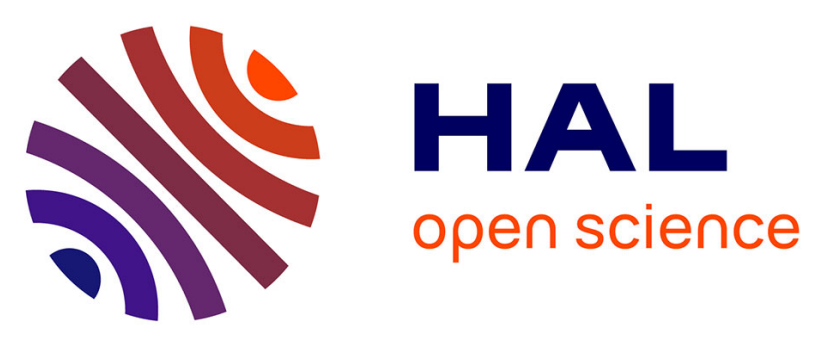

\title{
Alzheimer pathology disorganizes cortico-cortical circuitry: direct evidence from a transgenic animal model
}

\author{
B. Delatour, V. Blanchard, L. Pradier, Charles Duyckaerts
}

\section{- To cite this version:}

B. Delatour, V. Blanchard, L. Pradier, Charles Duyckaerts. Alzheimer pathology disorganizes corticocortical circuitry: direct evidence from a transgenic animal model. Neurobiol Dis, 2004, 16 (1), pp.41-7. 10.1016/j.nbd.2004.01.008 . hal-03236866

\section{HAL Id: hal-03236866 \\ https://cnrs.hal.science/hal-03236866}

Submitted on 26 May 2021

HAL is a multi-disciplinary open access archive for the deposit and dissemination of scientific research documents, whether they are published or not. The documents may come from teaching and research institutions in France or abroad, or from public or private research centers.
L'archive ouverte pluridisciplinaire HAL, est destinée au dépôt et à la diffusion de documents scientifiques de niveau recherche, publiés ou non, émanant des établissements d'enseignement et de recherche français ou étrangers, des laboratoires publics ou privés. 


\title{
Alzheimer pathology disorganizes cortico-cortical circuitry:
}

\section{Direct evidence from a transgenic animal model}

\author{
Delatour $^{1,2, \#}$, B; Blanchard ${ }^{3}$, V; Pradier $^{3}$, L; Duyckaerts ${ }^{2}$, C
}

${ }^{\#}$ Corresponding author

${ }^{1}$ Laboratoire de Neurobiologie de l'Apprentissage, de la Mémoire \& de la Communication, NAMC, CNRS UMR 8620, Bât 446, Université Paris-Sud, 91405 Orsay Cedex, FRANCE.

Tel: 33169154988

Fax: 33169157726

Email: benoit.delatour@ibaic.u-psud.fr

${ }^{2}$ Laboratoire de Neuropathologie R Escourolle, Inserm U106 and IFR Neurosciences, Groupe Hospitalier Pitié-Salpêtrière, Université Paris VI 47 Bd de l'Hôpital, 75651 Paris, Cedex 13, FRANCE.

${ }^{3}$ Aventis Pharma, Neurodegenerative Disease Group, Centre de Recherche de Paris, B.P. 14, 94403 Vitry-sur-Seine Cedex, FRANCE. 


\section{Abstract}

It has been proposed that Alzheimer disease (AD) is associated with a "disconnection syndrome" due to the gradual loss of morphological and functional integrity of corticocortical pathways. This hypothesis derives from indirect neuropathological observations but, definitive evidence that AD primarily targets cortico-cortical networks is still lacking. By means of neuroanatomical anterograde tracing methods we have investigated, in a murine transgenic model of $\mathrm{AD}$, the impact of the amyloid burden on axonal terminals in different neural systems. Axonal tracings revealed, in accordance with the "disconnection syndrome" hypothesis, that cortico-cortical fibers are significantly disorganised. Terminal fields in local and distant cortical areas contained numerous swollen dystrophic neurites, often grouped in grape-like clusters, at the plaque periphery. In contrary to fibers of cortical origin, those originating from subcortical brain structures only showed limited signs of degeneration upon reaching their cortical targets. These observations suggest a selective disruption of corticocortical connections induced by AD brain pathology.

KEYWORDS: Alzheimer disease, connections, neurodegeneration, dystrophic neurites, amyloidosis, axonal tracing, cortex, transgenic mice, amyloid protein precursor, presenilin. 


\section{Introduction}

Pathological hallmarks of Alzheimer disease (AD) are classified into two types: extracellular (deposition of $\mathrm{A} \beta$ peptide, which may become amyloid) and intracellular (neurofibrillary alterations associated with accumulation of hyperphosphorylated tau protein). Senile plaques are composite lesions involving a dense core of $A \beta$ peptide surrounded by a crown of abnormal, sometimes tau-positive, neuritic processes. Studying the mechanisms of senile (neuritic) plaque formation may help understand how amyloidogenesis and neurofibrillary lesions are related. Degenerated fibers that innervate senile plaques are mainly axons; they, indeed, contain presynaptic vesicles (Terry et al., 1964) and neurofilament epitopes (Schmidt et al., 1991). A subset of these fibers are immunostained by ubiquitin and were originally classified as dystrophic because of their markedly altered morphology (Dickson 1997). The impact of axon pathology in neurodegenerative diseases has been somewhat underestimated (Coleman and Perry 2002) despite it being obvious that degenerated neurites may seriously hamper brain connectivity and, hence, have functional consequences (Knowles et al., 1999). The pathogenic importance of dystrophic neurites in AD has hence been repeatedly emphasized (Arai et al., 1990; Masliah et al., 1991; McKee et al., 1991; Mirra et al., 1991). Moreover, neuropathological data suggest that AD is associated with a cortical "disconnection syndrome" in which cortico-cortical pathways gradually loose their morphological and functional integrity (for reviews, see Arendt 2001; De Lacoste and White 1993).

If $\mathrm{AD}$ pathology involves connective alterations at the level of brain circuitries, are all neuronal pathways disrupted? If not, what is the topography of affected connections? To identify the anatomical origin of the processes associated with senile plaques, we carried out experimental studies in a transgenic mouse model based on the overexpression of human mutated genes (coding for the amyloid precursor protein - APP and for the presenilin 1 
protein -PS1) found in familial AD. This model reproduces some of the key neuropathological features of the disease. Accelerated $\mathrm{A} \beta$ deposition in $\mathrm{AD}$ transgenic models is the major, but not unique histological lesion (Price et al., 1998). In the vicinity of $\mathrm{A} \beta$ deposits, several neurodegenerative changes can be observed including dystrophic neurites (Masliah et al., 2001; Masliah et al., 1996; Sturchler-Pierrat et al., 1997).

In the present study, experiments were carried out using an APPxPS1 model that develops A $\beta$ deposits, in close association with neuritic alterations, at 2-3 months of age (Blanchard et al., 2003; Wirths et al., 2001). We applied in vivo neuronal tracing protocols to study brain connectivity in these genetically modified mice. To our knowledge, this latter approach has been taken in only one previous study in which the authors mapped the perforant pathway by injecting a tracer into the entorhinal cortex (Phinney et al., 1999). They provided evidence of abnormal, ectopic terminals at the level of the hippocampus (dentate gyrus) and the thalamus. Our goal was to determine the extent to which cortico-cortical connections were altered in relation with amyloid pathology. For a comparative approach we investigated the morphology of other fiber tracts, namely subcortical-cortical connections.

Preliminary reports on these findings have been published in abstract form (Delatour et al., 2002). 


\section{Materials and Methods}

\section{Transgenic mice}

A double thy1 APP751 SL (Swedish mutation KM670/671NL, London mutation V717I introduced in the human APP751 sequence) x HMG PS1 M146 L transgenic mouse line was generated and established in accordance with standard procedures using CBA/C57B16 hybrids as described elsewhere (Blanchard et al., 2003; Wirths et al., 2001). Ten aged (between 14 and 24 months) male $(n=4)$ and female $(n=6)$ APPxPS1 transgenic mice were used for the present study.

\section{Neuroanatomical tracings and immunohistochemistry}

Biotinylated dextran amine-BDA (Brandt and Apkarian 1992; Reiner et al., 1993; Veenman et al., 1992) was used as an anterograde tracer to visualize axonal tracts and normal or dystrophic terminal boutons. BDA is also transported retrogradely raising the possibility that collateral axons from retrogradely labeled neurons could contaminate the preparation. However, to minimize the impact of reverse transport, fine micropipettes were used (Reiner et al., 1993).

Injections of BDA (Molecular Probes, Eugene, USA, 10\% in $0.01 \mathrm{M}$ phosphate buffer - PB) were placed in the cortex (posterior cingulate cortex, $n=1$ mouse injected ; visual cortex, $n=2$ and hippocampus, $n=1$ ) or subcortical nuclei (septum, $n=3$ and thalamus, $n=3$ ). BDA injections in cortical and subcortical areas were equally distributed in male and female transgenic mice. Results from subsequent tracing studies did not show any noticeable effect of gender. A pilot study was previously performed in six non-transgenic mice to determine parameters for the injections in selected anatomic targets (stereotaxic coordinates, volume of tracer to be injected). No neuritic dystrophy was found in those control animals. 
Mice were anesthetized with a mixture of 4:3 ketamine-xylazine. BDA was stereotaxically injected using a glass micropipette (inner diameter: $15 \mu \mathrm{m}$ ), using coordinates adapted from the Franklin and Paxinos atlas (Franklin and Paxinos 1997). Tracer was iontophoresed by applying a positive pulsed DC current ( $6 \mu \mathrm{A} ; 7 \mathrm{~s}$ on / $7 \mathrm{~s}$ off) for 10-15 min. After a survival time of 7-10 days, animals were sacrificed with an overdose of sodium pentobarbital and were perfused transcardially with a fixative solution containing 4\% paraformaldehyde in $125 \mathrm{mM}$ PB. After removal, the brains were postfixed for one hour in the same fixative used for perfusion. Brains were stored overnight in a solution of $20 \%$ glycerin and $2 \%$ dimethylsulfoxide in $125 \mathrm{mM}$ PB for cryoprotection. Brains were subsequently sectioned (frontal $40 \mu \mathrm{m}$-thick sections) on a freezing microtome, collected and rinsed with PB $(125 \mathrm{mM})$. Sections were then washed in a $0.05 \mathrm{M}$ triton X-100 saline phosphate buffer (PBS) solution and incubated, at room temperature, with an avidin-biotin-peroxidase complex (ABC Vectastain standard kit, Vector Laboratories, Burlingame, USA, 1:400) for $90 \mathrm{~min}$. The following reaction made use of nickel-enhanced diaminobenzidine, which precipitated as a gray/dark product. In a second step, labeling of amyloid plaques was done by standard Congo red staining (30 min in a $80 \%$ ethanol solution saturated with congo red and sodium chloride ; adapted from Puchtler et al., 1962) or by A $\beta$ immunohistochemistry. For the latter method, we used polyclonal antibodies directed against $A \beta$ 1-40 or A $\beta$ 1-42 (FCA-3340 and FCA3542, gift from F. Checler, dilution: 1/500) that were revealed using peroxidase-antiperoxidase (PAP) reaction rather than avidin-biotin-peroxidase method. This method was chosen to avoid cross-reactions with the biotin residue of the tracer. The chromogen used to visualize $\mathrm{A} \beta$ was the diaminobenzidine which precipitates as a brown product. Synaptophysin immunostaining was carried out to determine the occurrence and morphology of axon terminals at the periphery of the plaques. A primary polyclonal anti-synaptophysin antibody (Dako, Glostrup, Denmark, 1:200) was used and detected with the PAP method. 
Degeneration of traced terminals was finally assessed using double (BDA/ubiquitin) fluorescent immunolabelings that were analyzed by means of a confocal laser-scanning microscope (TCS SP1, Leica, Rueil Malmaison, France). BDA was revealed using a streptavidin-cyanine 2 complex (Jackson Immunoresearch, West Grove, USA, dilution: 1:200). Ubiquitin was detected with a polyclonal antibody (Dako, 1:500) and subsequently a goat anti-rabbit secondary antibody conjugated cyanine 3 (Jackson Immunoresearch, dilution: $1: 1000)$. 


\section{Results}

\section{Axon dystrophic terminals}

All injection sites were located in plaque-enriched (cortical and subcortical) areas. In each case a high density of abnormally enlarged neurites containing the anterograde tracer was observed, close to the injection site, surrounding amyloid plaques (Figure 1-A). These observations indicate that local circuitries, involving short axonal projections, were affected in both cortical and subcortical structures. In regard to long distance connections, only a subset of traced projections showed morphologically altered terminals around amyloid deposits (see below).

In both cortical and subcortical brain areas, swollen distended neurites were often observed to coalesce in grape-like clusters around the plaques. These were made of a dense core of Congo red positive material strongly immunoreactive to $A \beta$ antibodies. Abnormal neurites were not found in diffuse deposits of $A \beta$ peptide i.e. those $>30 \mu \mathrm{m}$ in diameter, Congo red negative and with indistinct contours. Double immunofluorescence indicated that most of BDA-traced puffy terminals showed ubiquitin labeling (see Figure 2-A,B,C), suggesting that they had undergone some degenerative processes.

In rare cases $(n=2)$, BDA injections induced retrograde labeling in neurons located in adjacent brain regions (e.g. in the parietal cortex following injection in the posterior cingulate cortex). In these cases neuronal cell bodies and their dendritic tree were filled, with a Golgi-like appearance (see Figure 1-B). The morphology of dendritic spines did not appear to be modified in the vicinity of the A $\beta$ deposits. On the contrary, synaptophysin immunostaining revealed a large number of synaptophysin-positive, distorted, enlarged axon terminals surrounding $\mathrm{A} \beta$ deposits (Figure 1-C). 


\section{Cortical projections}

BDA was injected at various cortical loci (eg Figure 2-D). Plaque-associated dystrophic neurites were noted close to the injection site (Figure 2-F) in all areas.

Projections from the associative visual cortex gave rise, at some distance, to pathological endings in the retrosplenial cortex (Figure 2-H). When the tracer was injected in the posterior cingulate cortex, terminals were found in various cortical targets that were either in the near vicinity of the injection site (eg presubiculum) or at a greater distance (eg dorsomedial prefrontal cortex). In all cases we found evidence of numerous pathological terminal fields around $\mathrm{A} \beta$ deposits. One mouse received an injection in the dorsal CA1 field of the hippocampus; direct projections, ending with abnormally dilated boutons, were observed in the plaque-enriched subicular complex.

To summarize, whatever target cortical area was considered, numerous BDA-positive dystrophic neurites were noted at the plaque periphery. Not only short cortico-cortical axons (such as CA1-subicular connections) but also long distance fiber association pathways (such as cingulo-frontal fibers that map the whole caudo-rostral extent of the brain to reach their targets) showed marked morphological alterations in relation to amyloid deposits. Hence, neuroanatomical tracing revealed that cortico-cortical fibers were significantly disorganised in the context of amyloid pathology, both at the level of intrinsic and extrinsic circuitries.

\section{Subcortical projections}

In other animals, the tracer was iontophoresed in different subcortical locations (eg Figure 2E). As mentioned above, we noticed that intrinsic connections, at the level of the injection site, were affected around plaques. Hence, when thalamic and septal nuclei were injected, a large number of dystrophic neurites were observed directly adjacent to the injection sites in 
these structures (Figure 2-G). Subcortical circuitries involving connections between distant structures were also disrupted. For example, after injections in the septal area, projections in the accumbens nucleus were associated with distorted enlarged terminals observed at the plaques' periphery.

While subcortical-subcortical connections were found to be morphologically altered in relation with $\mathrm{A} \beta$ deposits, we found no evidence that a similar process occurred for projections terminating in cortical areas. In all examined cases only a very small number of dystrophic neurites were noticed at the level of cortical axonal terminal fields. For instance, despite very dense projections from the mediodorsal thalamic nucleus to the orbital and dorsomedial prefrontal cortices, no clear evidence of pathological boutons were seen close to the amyloid deposits in these cortical regions (Figure 2-I). Furthermore, only rare dystrophic terminals were noted at the level of thalamo-insular or septo-hippocampal connections (Figure 3).

To summarize, as opposed to cortico-cortical connections, fibers originating in subcortical brain structures, did not show obvious signs of neurodegeneration when reaching their cortical targets. However, fibers remaining in subcortical networks presented pathological endings associated with neighbouring $\mathrm{A} \beta$ plaques. 


\section{Discussion}

By means of tracing experiments we provided direct confirmation of plaque-associated axonal dystrophy in APPxPS1 mice. Traced axons were abnormal both at the morphological (enlarged boutons) and immunohistochemical (labeling with antibodies raised against ubiquitin) levels (see also Masliah et al., 1996).

Terminal endings in dystrophic neurites surrounding plaques were evidenced by their immunoreactivity for synaptophysin. These observations, pointing to the presence of synaptic vesicles in dystrophic neurites, replicate previous observations obtained in the same transgenic line (Blanchard et al., 2003) as well as in other mouse models (Phinney et al., 1999) and in AD brains (Masliah et al., 1991). Electron microscopy has also provided strong evidence that synaptic elements are present in dystrophic neurites in both humans and genetically modified mice (Phinney et al., 1999; Terry et al., 1964).

In the present study, short and long cortico-cortical axons were disrupted at the plaque periphery. Intrinsic circuitries within cortical or subcortical areas underwent a similar neurodegenerative process. However, striking differences were seen between cortical and subcortical afferent fibers to cortical plaques. For instance, while cingulate cortical projections degenerated in the frontal cortex, thalamic fibers terminating in the same area showed no obvious sign of axonal pathology despite heavy amyloid burden. Interestingly, human neuropathological data also indicate that there is no evidence of a thalamic innervation of cortical seniles plaques (Duyckaerts et al., 1986). Extending previous observations (Phinney et al., 1999), our results support the hypothesis that neurites of cortical senile plaques are mainly axons from adjacent or distal cortical areas. No clear evidence was found that these neurites could have a subcortical origin. We recently immunolabeled catecholaminergic fibers (using tyrosine hydroxylase immunohistochemistry) in the cortex of 
APPxPS1 mice to evaluate morphological changes in cortical axon terminals arising from cell bodies located in subcortical nuclei. Once again we failed to demonstrate high number of dystrophic neurites associated with cortical plaques (unpublished observations). Supporting these observations, Cervera and collaborators (1990) also showed the lack of tyrosine hydroxylase positive senile plaques in the neocortex of $\mathrm{AD}$ patients.

This selective involvement of cortico-cortical fibers in plaque-associated neuritic degeneration can be paralleled with neuroimaging data obtained in human subjects. MRI studies have demonstrated, in $\mathrm{AD}$ patients, disruptions of connections linking cortical areas, as revealed by callosal atrophy (Hampel et al., 1998; Teipel et al., 2002). Similarly, functional brain imaging studies (using PET-based functional connectivity mapping or EEG coherence recordings) indicate alterations of cortico-cortical connections (e.g. between prefrontal and medial temporal lobe regions) in AD patients (Grady et al., 2001; Leuchter et al., 1992). Dementia severity has been correlated with the degree of callosal atrophy (Hampel et al., 1998; Teipel et al., 2002), suggesting that hodological alterations during the course of AD might lead to a disconnection syndrome with clinical (disrupted cognitive functioning) consequences.

Our observations in the APPxPS1 transgenic model, suggest that vulnerability of neuronal connections is selective. While some fiber systems are affected (e.g. cortico-cortical and subcortico-subcortical connections) others are largely preserved (e.g. subcortico-cortical connections). Several factors could explain this differential vulnerability. The amyloid cascade hypothesis would predict that axonal dystrophy develops within neuronal processes in response to $\mathrm{A} \beta$ aggregation. In accordance with this hypothesis, dystrophic, degenerated (ubiquitine positive) neurites are found at 2-3 months of age in the transgenic line used here (Blanchard et al., 2003) when A $\beta$ deposition starts. They develop in parallel with A $\beta$ 
deposition. $A \beta$ peptide could act as a trophic substance to trigger pathological sprouting of afferent fibers (Geddes et al., 1986; Masliah et al., 1991) or conversely might have direct neurotoxic effects (Koh et al., 1990; Neve and Robakis 1998; Yankner et al., 1989). While numerous data indicate a detrimental effect of $\mathrm{A} \beta$, as well as other plaque-associated peptides on neuronal cells and processes (Forloni et al., 2000; Marcon et al., 1999; Yang et al., 2000), it remains to be determined how $A \beta$ could alter selectively distinct axon populations. Differential vulnerability could depend on neurochemical properties of neural connections. Glutamatergic pathways, that are mainly involved in cortico-cortical projections, have been repeatedly reported to be altered (at both pre- and postsynaptic levels) in AD brains (Greenamyre and Young 1989; Lee et al., 2002; Myhrer 1993). Neuropeptidergic systems have also been shown to be differentially affected, in relation to their excitatory/inhibitory properties, in the brain of transgenic mice (Diez et al., 2000). According to another hypothesis, selective vulnerability of neural pathways is related to the content of neurofilaments and calcium-binding proteins in the sensitive neurons (Hof et al., 1990; Morrison et al., 1998) or to the degree of axon myelination (Braak et al., 2000). Disruption of cortico-cortical fibers might also rely on the specific topographical distribution of $\mathrm{A} \beta$ deposits: Armstrong and collaborators (2001) recently demonstrated that senile plaques in AD brains are evenly spaced in clusters paralleling the organisation of feedforward and feedback cortico-cortical pathways. This regular, non-random distribution of $A \beta$ deposits could therefore determine degeneration of selected neural pathways.

Finally, a combination of the different factors that we have considered might explain why degenerating neurites are principally axons, and why only some axons degenerate. Both plaque properties (local factors differentially expressed in amyloid deposits depending on cerebral topography) and morphological characteristics of axon populations (related to their anatomical origin) could interact and promote neurodegenerative effects. 
Further research effort is required to untangle this multicausal process and to find out how dystrophic neurites (as well as other neurofibrillary alterations) are related to $A \beta$ aggregation . Interestingly, it has been shown that $A \beta$ injection in the hippocampus of transgenic mice overexpressing P301L mutant human tau retrogradely promotes tangle formation in neurons of brain regions that project to the locus of injection (Gotz et al., 2001). This suggests that neural connections could be a substrate of interaction between $\mathrm{A} \beta$ deposits and tau-positive neurofibrillary lesions.

Both $A \beta$ deposition and neuritic alterations compromise the integrity of brain circuitry. A treatment targeting abnormal $A \beta$ metabolism and deposition should also prevent the lesions of the connective network in order to have clinical impact. 


\section{Figure legends}

\section{Figure 1}

A: Innervated plaques, at a short distance from the injection site, in the visual cortex of an APPxPS1 mouse. The amyloid deposits (stars) were immunostained with an anti-A $\beta$ antibody. BDA-positive enlarged terminals (black) were observed at the periphery of plaques (see arrows).

B: Retrogradely labeled pyramidal cells in the parietal cortex (black). Note that the dendritic trees were not altered in the vicinity of $\mathrm{A} \beta$ deposits (indicated by stars).

C: Synaptophysin immunoreactivity in the cortex of an APPxPS1 mouse. Abnormally enlarged synaptophysin-positive terminals (arrow heads) were constantly observed around A $\beta$ deposits (stars).

Scale bars: $50 \mu \mathrm{m}$

\section{Figure 2}

A-C: Colocalisation of BDA (A) and ubiquitin (B) in the same puffy axon terminals is illustrated in the merged photograph $(\mathrm{C})$. In some dilated neurites, the tracer BDA was present and colocalized with ubiquitin (arrows), while non-traced dystrophic terminals contained only ubiquitin (arrow heads). Neurites were in contact with a deposit of A $\beta$ peptide (star).

Confocal microscopy: BDA was visualized using a streptavidin-CY2 complex (red channel) and ubiquitin by a conjugated CY3 complex (green channel).

D: Example of cortical injection site (visual cortex)

E: Example of subcortical injection site (mediodorsal thalamic nucleus) 
F: Intrinsic cortico-cortical connections. The tracer was injected in the visual cortex; shortdistance axonal projections (black) ending in the same cortical area showed marked morphological alterations (arrows) around amyloid deposits (brown).

G: Intrinsic subcortico-subcortical connections. BDA was iontophoresed in the thalamus and local projections (black) showed signs of dystrophy (arrows) at the periphery of thalamic amyloid plaques (stained with Congo red).

H: Extrinsic cortico-cortical connections. The tracer was injected in the visual cortex and terminal fields were observed in the retrosplenial cortex. Note the presence of numerous BDA positive enlarged terminals (black; see arrows) around $\mathrm{A} \beta$ deposits (brown).

I: Subcortico-cortical connections. Thalamo-prefrontal projections (black) were traced after BDA injection in the mediodorsal nucleus of the thalamus. No obvious signs of axonal dystrophy were observed in contact with prefrontal $A \beta$ plaques (brown). Inset in the right upper corner shows at higher magnification the area delimited by dotted outlines (compare with figure 1-A).

Scale bars: $10 \mu \mathrm{m}(\mathrm{A}-\mathrm{C})$ and $50 \mu \mathrm{m}(\mathrm{D}-\mathrm{I})$.

\section{Figure 3}

Illustration of septo-hippocampal fibers. The tracer was injected in septal nuclei and terminal fields were observed in the hippocampus. Note the absence of dystrophic terminals at the plaque (star) periphery.

Scale bar: $25 \mu \mathrm{m}$ 


\section{Acknowledgements}

This work was supported by the Aventis Pharma ${ }^{\circledR}$ Alzheimer network and by the Fondation pour la Recherche Médicale.

We thank Jean-Jacques Hauw for stimulating discussions and continuous encouragements.

We are grateful to Frédéric Checler for the gracious gift of the FCA-3340 and FCA-3542

antibodies.

The help of H. El Hachimi for histological work is greatly acknowledged. Our appreciation

goes to J. Horwood for her indispensable aid in the redaction of the manuscript. 


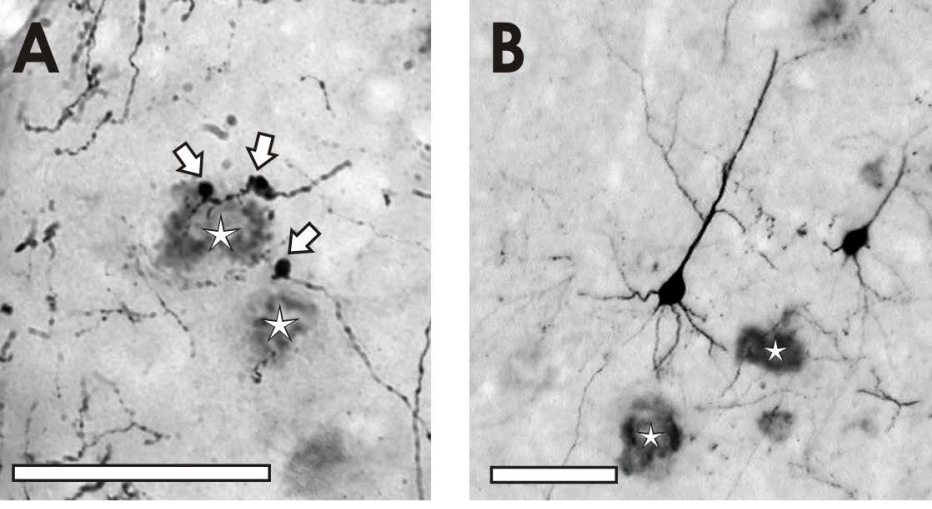




\section{References}

Arai, H., Lee, V.M., Otvos, L., Jr., Greenberg, B.D., Lowery, D.E., Sharma, S.K., Schmidt, M.L. \& Trojanowski, J.Q. (1990) Defined neurofilament, tau, and beta-amyloid precursor protein epitopes distinguish Alzheimer from non-Alzheimer senile plaques. Proc. Natl. Acad. Sci. USA, 87, 2249-2253.

Arendt, T. (2001) Alzheimer's disease as a disorder of mechanisms underlying structural brain self-organization. Neuroscience, 102, 723-765.

Armstrong, R.A., Lantos, P.L. \& Cairns, N.J. (2001) The spatial patterns of prion protein deposits in Creutzfeldt-Jakob disease: comparison with beta-amyloid deposits in Alzheimer's disease. Neurosci. Lett., 298, 53-56.

Blanchard, V., Moussaoui, S., Czech, C., Touchet, N., Bonici, B., Planche, M., Canton, T., Jedidi, I., Gohin, M., Wirths, O., Bayer, T.A., Langui, D., Duyckaerts, C., Tremp, G. \& Pradier, L. (2003) Time Sequence of Maturation of Dystrophic Neurites Associated with A $\beta$ Deposits in APP/PS1 Transgenic Mice. Exp. Neurol., 184, 247-263.

Braak, H., Del Tredici, K., Schultz, C. \& Braak, E. (2000) Vulnerability of select neuronal types to Alzheimer's disease. Ann. N. Y. Acad. Sci., 924, 53-61.

Brandt, H.M. \& Apkarian, A.V. (1992) Biotin-dextran: a sensitive anterograde tracer for neuroanatomic studies in rat and monkey. J. Neurosci. Methods, 45, 35-40.

Cervera, P., Duyckaerts, C., Ruberg, M., Hirsch, E., Ransmayr, G., Hauw, J.J. \& Agid, Y. (1990) Tyrosine hydroxylase-like immunoreactivity in senile plaques is not related to the density of tyrosine hydroxylase-positive fibers in patients with Alzheimer's disease. Neurosci. Lett., 110, 210-215.

Coleman, M.P. \& Perry, V.H. (2002) Axon pathology in neurological disease: a neglected therapeutic target. Trends Neurosci., 25, 532-537. 
De Lacoste, M.C. \& White, C.L., 3rd (1993) The role of cortical connectivity in Alzheimer's disease pathogenesis: a review and model system. Neurobiol. Aging, 14, 1-16.

Delatour, B., Blanchard, V., Pradier, L. \& Duyckaerts, C. (2002) Alzheimer's pathology disrupts the morphology of selected brain connections. In: 3rd Forum of European Neuroscience, Paris, France.

Dickson, D.W. (1997) The pathogenesis of senile plaques. J. Neuropathol. Exp. Neurol., 56, 321-339.

Diez, M., Koistinaho, J., Kahn, K., Games, D. \& Hokfelt, T. (2000) Neuropeptides in hippocampus and cortex in transgenic mice overexpressing V717F beta-amyloid precursor protein--initial observations. Neuroscience, 100, 259-286.

Duyckaerts, C., Hauw, J.-J., Bastenaire, F., Piette, F., Poulain, C., Rainsard, V., Javoy-Agid, F. \& Berthaux, P. (1986) Laminar distribution of neocortical plaques in senile dementia of the Alzheimer type. Acta. Neuropathol. (Berlin), 70, 249-256.

Forloni, G., Bertani, I., Calella, A.M., Thaler, F. \& Invernizzi, R. (2000) Alpha-synuclein and Parkinson's disease: selective neurodegenerative effect of alpha-synuclein fragment on dopaminergic neurons in vitro and in vivo. Ann. Neurol., 47, 632-640.

Franklin, K.B.J. \& Paxinos, G. (1997) The mouse brain in stereotaxic coordinates. Academic Press, San Diego.

Geddes, J.W., Anderson, K.J. \& Cotman, C.W. (1986) Senile plaques as aberrant sproutstimulating structures. Exp. Neurol., 94, 767-776.

Gotz, J., Chen, F., van Dorpe, J. \& Nitsch, R.M. (2001) Formation of neurofibrillary tangles in P3011 tau transgenic mice induced by Abeta 42 fibrils. Science, 293, 1491-1495.

Grady, C.L., Furey, M.L., Pietrini, P., Horwitz, B. \& Rapoport, S.I. (2001) Altered brain functional connectivity and impaired short-term memory in Alzheimer's disease. Brain, 124, 739-756. 
Greenamyre, J.T. \& Young, A.B. (1989) Excitatory amino acids and Alzheimer's disease. Neurobiol. Aging, 10, 593-602.

Hampel, H., Teipel, S.J., Alexander, G.E., Horwitz, B., Teichberg, D., Schapiro, M.B. \& Rapoport, S.I. (1998) Corpus callosum atrophy is a possible indicator of region- and cell type-specific neuronal degeneration in Alzheimer disease: a magnetic resonance imaging analysis. Arch Neurol, 55, 193-198.

Hof, P.R., Cox, K. \& Morrison, J.H. (1990) Quantitative analysis of a vulnerable subset of pyramidal neurons in Alzheimer's disease: I. Superior frontal and inferior temporal cortex. J. Comp. Neurol., 301, 44-54.

Knowles, R.B., Wyart, C., Buldyrev, S.V., Cruz, L., Urbanc, B., Hasselmo, M.E., Stanley, H.E. \& Hyman, B.T. (1999) Plaque-induced neurite abnormalities: implications for disruption of neural networks in Alzheimer's disease. Proc. Natl. Acad. Sci. USA, 96, $5274-5279$.

Koh, J.Y., Yang, L.L. \& Cotman, C.W. (1990) Beta-amyloid protein increases the vulnerability of cultured cortical neurons to excitotoxic damage. Brain. Res., 533, 315320.

Lee, H.G., Zhu, X., Ghanbari, H.A., Ogawa, O., Raina, A.K., O'Neill, M.J., Perry, G. \& Smith, M.A. (2002) Differential regulation of glutamate receptors in Alzheimer's disease. Neurosignals, 11, 282-292.

Leuchter, A.F., Newton, T.F., Cook, I.A., Walter, D.O., Rosenberg-Thompson, S. \& Lachenbruch, P.A. (1992) Changes in brain functional connectivity in Alzheimer-type and multi- infarct dementia. Brain, 115, 1543-1561.

Marcon, G., Giaccone, G., Canciani, B., Cajola, L., Rossi, G., De Gioia, L., Salmona, M., Bugiani, O. \& Tagliavini, F. (1999) A betaPP peptide carboxyl-terminal to Abeta is neurotoxic. Am. J. Pathol., 154, 1001-1007. 
Masliah, E., Mallory, M., Hansen, L., Alford, M., Albright, T., DeTeresa, R., Terry, R., Baudier, J. \& Saitoh, T. (1991) Patterns of aberrant sprouting in Alzheimer's disease. Neuron, 6, 729-739.

Masliah, E., Sisk, A., Mallory, M. \& Games, D. (2001) Neurofibrillary pathology in transgenic mice overexpressing V717F beta-amyloid precursor protein. $J$. Neuropathol. Exp. Neurol., 60, 357-368.

Masliah, E., Sisk, A., Mallory, M., Mucke, L., Schenk, D. \& Games, D. (1996) Comparison of neurodegenerative pathology in transgenic mice overexpressing V717F betaamyloid precursor protein and Alzheimer's disease. J. Neurosci., 16, 5795-5811.

McKee, A.C., Kosik, K.S. \& Kowall, N.W. (1991) Neuritic pathology and dementia in Alzheimer's disease. Ann. Neurol., 30, 156-165.

Mirra, S.S., Heyman, A., McKeel, D., Sumi, S.M., Crain, B.J., Brownlee, L.M., Vogel, F.S., Hughes, J.P., van Belle, G. \& Berg, L. (1991) The Consortium to Establish a Registry for Alzheimer's Disease (CERAD). Part II. Standardization of the neuropathologic assessment of Alzheimer's disease. Neurology, 41, 479-486.

Morrison, B.M., Hof, P.R. \& Morrison, J.H. (1998) Determinants of neuronal vulnerability in neurodegenerative diseases. Ann. Neurol., 44, S32-44.

Myhrer, T. (1993) Animal models of Alzheimer's disease: glutamatergic denervation as an alternative approach to cholinergic denervation. Neurosci Biobehav Rev, 17, 195-202.

Neve, R.L. \& Robakis, N.K. (1998) Alzheimer's disease: a re-examination of the amyloid hypothesis. Trends Neurosci., 21, 15-19.

Phinney, A.L., Deller, T., Stalder, M., Calhoun, M.E., Frotscher, M., Sommer, B., Staufenbiel, M. \& Jucker, M. (1999) Cerebral amyloid induces aberrant axonal sprouting and ectopic terminal formation in amyloid precursor protein transgenic mice. J. Neurosci., 19, 8552-8559. 
Price, D.L., Tanzi, R.E., Borchelt, D.R. \& Sisodia, S.S. (1998) Alzheimer's disease: genetic studies and transgenic models. Annu Rev Genet, 32, 461-493.

Puchtler, H., Sweat, F. \& Levine, M. (1962) On the binding of Congo red by amyloid. J. Histochem. Cytochem., 10, 355-364.

Reiner, A., Veenman, C.L. \& Honig, M.G. (1993) Anterograde tracing using biotinylated dextran amine. In: Neuroscience Protocols (ed. by F.G. Wouterlood). Elsevier, Amsterdam.

Schmidt, M.L., Lee, V.M. \& Trojanowski, J.Q. (1991) Comparative epitope analysis of neuronal cytoskeletal proteins in Alzheimer's disease senile plaque neurites and neuropil threads. Lab. Invest., 64, 352-357.

Sturchler-Pierrat, C., Abramowski, D., Duke, M., Wiederhold, K.H., Mistl, C., Rothacher, S., Ledermann, B., Burki, K., Frey, P., Paganetti, P.A., Waridel, C., Calhoun, M.E., Jucker, M., Probst, A., Staufenbiel, M. \& Sommer, B. (1997) Two amyloid precursor protein transgenic mouse models with Alzheimer disease-like pathology. Proc. Natl. Acad. Sci. USA, 94, 13287-13292.

Teipel, S.J., Bayer, W., Alexander, G.E., Zebuhr, Y., Teichberg, D., Kulic, L., Schapiro, M.B., Moller, H.J., Rapoport, S.I. \& Hampel, H. (2002) Progression of corpus callosum atrophy in Alzheimer disease. Arch Neurol, 59, 243-248.

Terry, R.D., Gonatas, J.K. \& Weiss, M. (1964) Ultrastructural studies in Alzheimer presenile dementia. Am. J. Pathol., 44, 269-297.

Veenman, C.L., Reiner, A. \& Honig, M.G. (1992) Biotinylated dextran amine as an anterograde tracer for single- and double-labeling studies. J. Neurosci. Methods, 41, 239-254. 
Wirths, O., Multhaup, G., Czech, C., Blanchard, V., Tremp, G., Pradier, L., Beyreuther, K. \& Bayer, T.A. (2001) Reelin in plaques of beta-amyloid precursor protein and presenilin1 double-transgenic mice. Neurosci. Lett., 316, 145-148.

Yang, F., Ueda, K., Chen, P., Ashe, K.H. \& Cole, G.M. (2000) Plaque-associated alphasynuclein (NACP) pathology in aged transgenic mice expressing amyloid precursor protein. Brain. Res., 853, 381-383.

Yankner, B.A., Dawes, L.R., Fisher, S., Villa-Komaroff, L., Oster-Granite, M.L. \& Neve, R.L. (1989) Neurotoxicity of a fragment of the amyloid precursor associated with Alzheimer's disease. Science, 245, 417-420. 